\title{
Integral charge quasiparticles in a fractional quantum Hall liquid
}

\author{
Giovanni Vignale* \\ Department of Physics and Astronomy, University of Missouri, Columbia, Missouri 65211, USA
}

(Received 18 October 2005; published 7 February 2006)

\begin{abstract}
Starting from a collective description of the incompressible fractional quantum Hall liquid as an elastic medium that supports gapped neutral excitations, I show that the one-electron spectral function of this system exhibits a sharp peak at the lowest available excitation energy, well separated from the continuum spectrum at higher energy. I interpret this peak as the signature of the integral charge quasiparticle recently predicted by Peterson and Jain [Phys. Rev. Lett. 94, 186808 (2005)] and calculate its spectral weight for different filling factors.
\end{abstract}

DOI: 10.1103/PhysRevB.73.073306

PACS number(s): 73.43.Lp, 73.43.Jn, 71.10.Hf

The fate of one-particle excitations in strongly correlated electronic systems is one of the most basic problems of many-body theory. Normal electron liquids in threedimensional (3D) and two-dimensional metals and semiconductors support one-particle excitations (quasiparticles) in spite of strong correlations. A one-dimensional electron liquid, on the other hand, does not support such quasiparticles. ${ }^{1}$

The two-dimensional electron liquid (2DEL) at a magnetic field so high that all the electrons reside in the lowest Landau level (LLL) (filling factor $\nu=N / N_{L}<1$, where $N_{L}$ is the degeneracy of the Landau level) is a perfect example of a system in which electron correlations lead to qualitatively new features. ${ }^{2}$ At filling factors of the form $\nu=n /(2 n p+1)$, with $n$ and $p$ integers, the electrons form an incompressible quantum liquid with fractionally charged excitations ${ }^{3,4}$ which obey unconventional statistics. ${ }^{5}$ At the same time, the neutral excitation spectrum exhibits a gap. ${ }^{6}$ This and other striking properties of the fractional quantum Hall states are now well understood in terms of the composite fermion $(\mathrm{CF})$ model. ${ }^{7,8}$

The one-electron properties of the 2DEL at high magnetic field have been intensively studied, both experimentally, through measurements of the tunneling density of states,,, 10 and theoretically, through analytical ${ }^{11,12}$ and numerical calculations $^{12-14}$ of the local spectral function. Neither the experiments nor the early calculations showed any evidence of the sharp peak in the tunneling density of states that would indicate the existence of a localized quasiparticle excitation of charge $e$. In a slightly later paper, Haussmann ${ }^{15}$ did find such a peak, but dismissed it as an artifact of the independent boson model on which his calculations (as well as the calculations of Ref. 11 and mine below) are based. It was tacitly assumed that integral charge quasiparticles must be absent in the fractional quantum Hall liquid.

Yet, the composite fermion theory of the incompressible quantum Hall liquid suggests a different picture in which single-particle excitations of integral charge $e$ can and do exist as bound states of composite fermions. ${ }^{17}$ Consider for example the incompressible liquid at filling factor $\nu=1 / 3$. In composite fermion theory, this corresponds to one full Landau level of composite fermions with two vortices attached to each: the $\mathrm{CF}$ filling factor is thus $\nu^{*}=1$. Let us remove three CFs from the fully occupied Landau level: it can be shown ${ }^{16}$ that the resulting $\mathrm{CF}$ wave function is just what one gets by applying the electron destruction operator to the ground state. We will refer to the state obtained in this manner as the "single-hole state." The perfect coincidence of the single-hole state with the state of three CF holes implies that the spectral function for removal of one electron has a single peak at the energy required to remove three CFs. This is very interesting because it suggests that a charge- $e$ hole can exist as a bound complex of three CF holes.

Recently, Jain and Peterson ${ }^{17}$ have extended the above analysis to the case of electron quasiparticles at $\nu=1 / 3$, as well as electron and hole quasiparticles at $\nu=2 / 5$ and $3 / 7$ (corresponding, respectively, to two and three full $\mathrm{CF}$ Landau levels). By carefully extrapolating the results of their calculations to the thermodynamic limit, they have found that electron and hole quasiparticles exist in all cases and can be identified with complex bound states of CFs in different CF-Landau levels. In each case, they find that the strength $Z$ of the quasielectron peak is smaller than the strength of the quasihole peak. Furthermore, the intensity of these peaks decreases rapidly as the size of the $q=0$ gap in the collective excitation spectrum decreases. This trend is consistent with the expectation that there should be no quasiparticles in the compressible state at filling factor $\nu=1 / 2$ - the gapless limit of the Jain's sequence. ${ }^{12,18}$ Ironically, it is precisely the presence of the quantum Hall gap - the most un-Fermi-liquidlike feature of the quantum Hall liquid - that "protects" the electron and the hole quasiparticles from total collectivization. Jain and Peterson ${ }^{17}$ have further pointed out that the existence of these quasiparticles could be observed in vertical tunneling experiments and discussed the reasons why they may have been missed in the experiments carried out so far.

The purpose of this report is to rederive the existence of integral charge electron (hole) quasiparticles in the LLL by what appears to be a completely different approach, since it does not make use of the notion of composite fermions. To this end, I present an approximate calculation of the oneelectron spectral function in the sector of zero angular momentum. At the absolute zero of temperature, this function splits into the sum of two nonoverlapping terms, one of which vanishes for negative frequency and the other for positive frequency. The positive-frequency part is defined as follows: 


$$
A_{>}(\omega)=\sum_{n}\left|\left\langle n\left|\hat{a}_{0}^{\dagger}\right| 0\right\rangle\right|^{2} \delta\left(\omega-\omega_{n 0}\right),
$$

where $\hat{a}_{0}^{\dagger}$ is the creation operator for the state of angular momentum $m=0$ (in the circularly symmetric gauge), the sum runs over the exact eigenstates $|n\rangle(|0\rangle$ being the ground state), and $\hbar \omega_{n 0}$ are excitation energies. The negativefrequency part $\left[A_{<}(\omega)\right]$ is obtained by replacing $\hat{a}_{0}^{\dagger}$ with $\hat{a}_{0}$ and $\omega$ with $-\omega$ in the above formula. I will show that this function contains a $\delta$-function peak at the lowest available excitation energy in the $m=0$ sector, and that this peak is well separated from the rest of the excitation spectrum-a fact already noticed by Haussmann ${ }^{15}$ and dismissed by him as an artifact of the "independent boson model." Since, by construction, the spectral function measures the distribution of charge- $\pm e$ excitations over exact eigenstates of the system, I believe that the split-off $\delta$-function peak should be interpreted as the signature of a long-lived quasiparticle of charge $\pm e$ - the Peterson-Jain quasiparticle. ${ }^{17}$ Notice that this quasiparticle has more energy than the "conventional" fractionally charged quasielectron or quasihole, but it can nevertheless be produced (if sufficient energy is available) with a probability proportional to the height of the peak. The process is analogous to the production of complex bound states of elementary particles in high energy physics.

One may ask why the peculiar structure of $A(\omega)$ described above was not noticed in earlier theoretical calculations of the spectral function. These calculations fall into two classes: exact diagonalizations of few-electron systems ${ }^{12,13}$ and approximate calculations for the infinite system. ${ }^{11,12}$ The former are exact but, by their very nature, produce an output that consists of a discrete set of spectral lines separated by gaps. It is therefore impossible to notice the presence of a single spectral line separated from a continuum, for the simple reason that there is no continuum. The analytical calculations, on the other hand, were based on approximate bosonization schemes in which the dispersion of the "bosons" was assumed to be gapless or, in one case, diffusive. The calculation presented below (as well as that of Haussmann ${ }^{15}$ ) is also based on an approximate bosonization scheme, but the crucial difference is that the bosons (which represent the neutral collective density oscillations of the liquid) are gapped. As I show below, the presence of the gap in the boson spectrum is the single feature that causes the appearance of the split-off peak in the spectral function.

Our starting point is the collective description of the quantum Hall liquid as an elastic medium that supports gapped collective excitation. ${ }^{19}$ The Hamiltonian (projected in the LLL) has the form

$$
\hat{H}_{0}=\sum_{\vec{q}} \hbar \omega_{q} \hat{b}_{\vec{q}}^{\dagger} \hat{b}_{\vec{q}}
$$

where $\omega_{q}$ is the frequency of the collective modes at wave vector $\vec{q}$, and we assume the dispersion has a gap, i.e., $\omega_{q}$ $>\omega_{\min }$ for all $\vec{q}$. The boson operators $\hat{b}_{\vec{q}}$ and $\hat{b}_{\vec{q}}^{\dagger}$ are related to the components of the elastic displacement field operator $\hat{\vec{u}}(\vec{r})$ in the following manner:

$$
\hat{\vec{u}}(\vec{r})=\frac{\ell}{\sqrt{n_{0} \mathcal{A}}} \sum_{\vec{q}}\left(\hat{b}_{\vec{q}} \vec{u}(\vec{q}) e^{i \vec{q} \cdot \vec{r}}+\hat{b}_{\vec{q}}^{\dagger} \vec{u}(\vec{q}) e^{-i \vec{q} \cdot \vec{r}}\right) .
$$

Here $n_{0}$ is the average density, $\ell=\sqrt{\hbar c / e B}$ is the magnetic length, $\mathcal{A}$ is the area of the system, and $\vec{u}(\vec{q})$ - the eigenfunction of the associated classical dynamical problem ${ }^{1}$ - is given by

$$
\vec{u}(\vec{q})=-i \vec{q} \ell \sqrt{\frac{\mathcal{S}}{2 \hbar \omega_{q} n_{0}}}+\frac{\vec{e}_{z} \times \vec{q}}{q^{2} \ell} \sqrt{\frac{\hbar \omega_{q} n_{0}}{2 \mathcal{S}}},
$$

where $\vec{e}_{z}$ is the unit vector perpendicular to the plane of the two-dimensional electron gas, and $\mathcal{S}$ is the dynamical shear modulus. Notice that, in order to have a collective dynamics, the medium must be assigned a finite shear modulus: this assignment is consistent with the liquid nature of the system as long as we work at finite frequencies, comparable to gap. Also notice that the bosonic commutation relation $\left[\hat{b}_{\vec{q}}, \hat{b}_{\vec{q}^{\prime}}^{\dagger}\right]$ $=\delta_{\vec{q} \vec{q}^{\prime}}$ is simply a way of saying that the $x$ and $y$ components of the projected displacement field are canonically conjugate variables: $\left[\hat{u}_{x}(\vec{r}), \hat{u}_{y}\left(\vec{r}^{\prime}\right)\right]=-i\left(\ell^{2} / n_{0}\right) \delta\left(\vec{r}-\vec{r}^{\prime}\right)$.

In order to investigate the possible existence of electron and hole quasiparticles, we consider the behavior of a single "test electron" added to the system in the coherent state of angular momentum $m=0$ in the LLL. This electron interacts with the density fluctuations of the medium via the Coulomb interaction, and since the density fluctuation is related to the displacement field by $\delta \hat{n}(\vec{r})=-n_{0} \vec{\nabla} \cdot \hat{\vec{u}}(\vec{r})$, we see that the complete Hamiltonian for the test electron plus the electron liquid has the form

$$
\hat{H}=\sum_{\vec{q}} \hbar \omega_{q} \hat{b}_{\vec{q}}^{\dagger} \hat{b}_{\vec{q}}+\sum_{\vec{q}} M_{q}\left(\hat{b}_{\vec{q}}+\hat{b}_{-\vec{q}}^{\dagger}\right) \hat{a}_{0}^{\dagger} \hat{a}_{0},
$$

where $M_{q}$ is an "electron-phonon matrix element," which, for Coulomb interaction, has the form

$$
M_{q}=-\frac{2 \pi e^{2} q \ell^{2}}{\epsilon_{b} \sqrt{\mathcal{A}}} \sqrt{\frac{\mathcal{S}}{2 \hbar \omega_{q}}} e^{-q^{2} \ell^{2} / 2} .
$$

It was shown in Refs. 1 and 19 that, for states described by the Laughlin wave function at filling factor $\nu=1 / M$, where $M$ is an odd integer, the dynamical shear modulus is related to the $q=0$ gap, $\Delta=\lim _{q \rightarrow 0} \hbar \omega_{q}$, by the simple formula

$$
\frac{\mathcal{S}}{n_{0} \Delta}=\frac{1-\nu}{4 \nu} .
$$

For different filling factors, we can either continue to use this relation or use the shear modulus of the classical Wigner crystal, which is given by $\mathcal{S} / n_{0}=0.0977 \sqrt{\nu}\left(e^{2} / \epsilon_{b} \ell\right) .{ }^{20}$

It should be noted at this point that the Hamiltonian $\hat{H}$ is formally similar to the polaron-problem Hamiltonian in which a free electron interacts with optical phonons. When the polaron Hamiltonian is generalized to describe a gas of free electrons interacting with collective density and spin fluctuations, it yields a pseudo-Hamiltonian which can be solved (to second order in the "electron-phonon interaction") leading to the standard theory of the normal Fermi liquid in 
3D, with long-lived electron and hole quasiparticles of effective mass $m^{*}{ }^{1,21}$ The present Hamiltonian has the crucial advantage that virtual transitions of the test electron to different single-particle states in the LLL are not allowed: the electron can never be scattered out of the $m=0$ state. It is therefore possible to obtain a complete analytic solution of the model Hamiltonian and to calculate the spectral function exactly.

It must also be noted that the form of the Hamiltonian (5) is such that only the spectral function of charge- $e$ excitations, i.e., the one-electron spectral function, can be studied with it. This is because the operator $\hat{a}_{0}^{\dagger}$ is the standard creation operator, which adds one electron to the ground state. Fractionally charged excitations cannot be created in this manner. Therefore, this method cannot be used to study the spectral distribution of fractionally charged quasiparticles.

The solution of the Hamiltonian (5) is well known. ${ }^{1}$ The electron-phonon coupling is eliminated by a unitary transformation. The new Hamiltonian (still written in terms of the original operators) takes the form

$$
\hat{\bar{H}}=\sum_{\vec{q}} \hbar \omega_{q} \hat{b}_{\vec{q}}^{\dagger} \hat{b}_{\vec{q}}+E_{p} \hat{a}_{0}^{\dagger} \hat{a}_{0},
$$

where $E_{p} \equiv-\Sigma_{\vec{q}} M_{q}^{2} / \hbar \omega_{q}$ is the polaron shift. $E_{p}$ can be calculated analytically if one approximates $\hbar \omega_{q} \simeq \Delta$, by making use of the Laughlin-wave-function-based expression (7) for $\mathcal{S} / n_{0} \Delta .{ }^{23}$ The result is

$$
E_{p}=-\frac{1-\nu}{16 \bar{\Delta}} \frac{e^{2}}{\epsilon_{b} \ell},
$$

(where $\bar{\Delta}$ is the gap $\Delta$ expressed in units of $e^{2} / \epsilon_{b} \ell$ ). Notice that $E_{p}$ is the addition energy of our model, i.e., the difference between the ground-state energies of the system with and without the "test electron." Its value is not expected to be quantitavely accurate since the elastic model is only valid at long wavelengths and certainly misses much of the shortrange correlations that control the value of the ground-state energy.

Let us now proceed to the calculation of the spectral function. A standard calculation leads to the following result for $A_{>}(\omega)$ :

$$
A_{>}(\omega)=\int_{-\infty}^{+\infty} \frac{d t}{2 \pi} e^{i\left(\omega-\omega_{p}\right) t+\sum_{\vec{q}}\left(M_{q} / \hbar \omega_{q}\right)^{2}\left(e^{-i \omega_{q} t}-1\right)},
$$

where $\omega_{p}=E_{p} / \hbar . A_{<}(\omega)$ is just the mirror image of $A_{>}(\omega)$ with respect to $\omega=0$.

A more effective way to write Eq. (10) is

$$
A_{>}(\omega)=Z \delta\left(\omega-\omega_{p}\right)+Z \sum_{k=1}^{\infty} \frac{g_{k}\left(\omega-\omega_{p}\right)}{k !},
$$

where

$$
Z=e_{\vec{q}}^{-\sum\left(M_{q} / \hbar \omega_{q}\right)^{2}},
$$

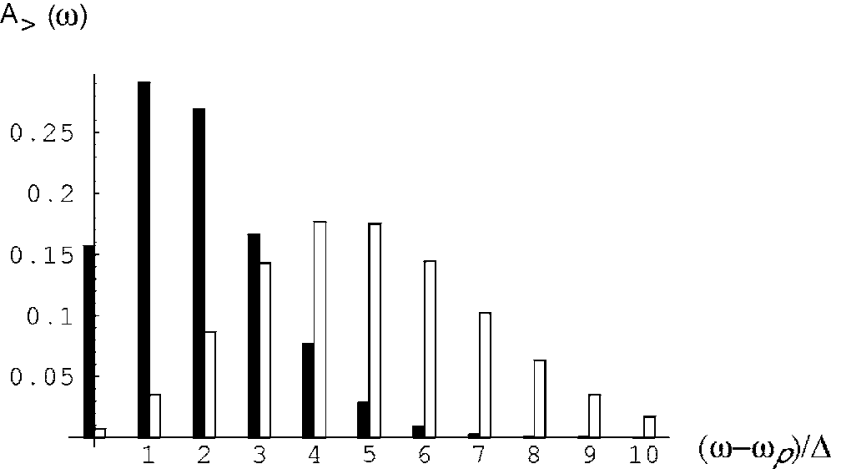

FIG. 1. The spectral function calculated from Eq. (15) at $\nu$ $=1 / 3, \bar{\Delta}=0.15$ (black bars) and $\nu=2 / 5, \bar{\Delta}=0.087$ (white bars).

$$
g_{1}(\omega)=\sum_{\vec{q}}\left(\frac{M_{q}}{\hbar \omega_{q}}\right)^{2} \delta\left(\omega-\omega_{q}\right)
$$

and

$$
g_{k}(\omega)=\int_{0}^{\infty} d \omega^{\prime} g_{1}\left(\omega-\omega^{\prime}\right) g_{k-1}\left(\omega^{\prime}\right),
$$

for $k>1$. From this, we see that the spectral function consists of two parts: a $\delta$-function peak at $\omega=\omega_{p}$ [first term on the right-hand side of Eq. (11)] and a continuum (second term). The continuum is a sum of terms $g_{k}\left(\omega-\omega_{p}\right) / k$ ! which can be evaluated recursively from Eqs. (13) and (14). The $k$ th term of this $\operatorname{sum}(k \geqslant 1)$ differs from zero in a range of frequencies going from $\omega_{p}+k \omega_{\min }$ to $\omega_{p}+k \omega_{\max }$, where $\omega_{\min }$ and $\omega_{\max }$ are the minimum and maximum values of $\omega_{\vec{q}}$. Assuming that the "bandwidth" of the collective mode, $\omega_{\max }-\omega_{\min }$ is larger than $\omega_{\min }$, we see that contributions with different $k$ overlap, resulting in a smooth variation of the spectral function for $\omega>\omega_{p}+\omega_{\min }$. However, the $\delta$ function at $\omega=\omega_{p}$ is split off, since $\omega_{p}$ is lower than $\omega_{p}+\omega_{\min }$. In other words, the presence of an isolated spectral line at $\omega=\omega_{p}$ is inescapable and independent of the details of the collective mode dispersion, as long as the latter has a gap. This is the quasiparticle peak. Its strength $Z$ is the probability of the electron entering the system in the lowest energy state, with all the magnetoplasmon oscillators remaining in their ground state. On the other hand, the area under the $k$ th term of the sum in Eq. (11) is the probability of the electron entering the system in an excited state containing $k$ quanta of the density oscillation field.

An analytical evaluation of Eq. (11) becomes possible if one neglects the dispersion of the density oscillations, setting $\omega_{q}=\Delta / \hbar$. The result is then

$$
A_{>}(\omega)=Z \sum_{k=0}^{\infty} \frac{1}{k !}\left(\frac{\left|E_{p}\right|}{\Delta}\right)^{k} \delta\left(\omega-k \Delta / \hbar-\omega_{p}\right),
$$

where

$$
Z=e^{-\left|E_{p}\right| \Delta} .
$$

In this approximation, and only in this approximation, the spectral function consists of a series of sharp spectral lines, as shown in Fig. 1. All these sharp lines are artifacts of the 
TABLE I. The dimensionless $q=0$ gap $\bar{\Delta}$, the dimensionless polaron shift $\bar{E}_{p}$, and the quasiparticle renormalization constant $Z$ for three different values of the filling factor $\nu . \bar{E}_{p}$ is $E_{p}$ in units of $e^{2} / \epsilon_{b} \ell$. The values of $\bar{\Delta}$ are from Ref. 22 .

\begin{tabular}{clcl}
\hline \hline$\nu$ & \multicolumn{1}{c}{$\bar{\Delta}$} & $\bar{E}_{p}$ & \multicolumn{1}{c}{$Z$} \\
\hline $1 / 3$ & 0.15 & -0.477 & 0.156 \\
$2 / 5$ & 0.087 & -0.431 & 0.007 \\
$3 / 7$ & 0.069 & -0.525 & 0.0004 \\
\hline \hline
\end{tabular}

dispersionless approximation, with the notable exception of the zero-phonon line, which, as discussed above, is present even when the dispersion of the collective modes is fully taken into account. Notice that the excitation probability attains its maximum when the total number of excitations is as close as possible to $E_{p} / \Delta$. As the gap ( $\Delta$ in the present approximation) tends to zero, the injected electron produces, on the average, larger and larger numbers of collective excitations: the strength of the zero-magnetoplasmon line tends to zero as the peak of the spectral function shifts to higher and higher excitation levels.

Table I shows the $q=0$ gap and the renormalization constant calculated from Eqs. (9) and (16) for three different values of $\nu=1 / 3$ to $2 / 5$ to $3 / 7$. The rapid decrease of $Z$ with decreasing gap is consistent with the trend observed by Jain and Peterson in their calculation. ${ }^{17}$ However, in contrast to that calculation, our model does not distinguish between the electron and the hole excitation. In linear elasticity theory, there is complete symmetry between these two types of excitations, since they do not differ in their long-range effect on the elastic medium.

It is remarkable that two very different approaches-the composite fermion theory and the continuum elasticity model-concur in predicting the possibility of charge- $e$ quasiparticles in the LLL. At a closer inspection, we see some similarities between the two approaches. In the composite fermion approach, the reduction of $Z$ arises from the fact that there are several excited states of composite fermions that have the same quantum numbers as the electron (on the other hand, when one removes one electron at $\nu=1 / 3$, there is only one $\mathrm{CF}$ bound state with the correct quantum numbers and this is why $Z=1$ in that case). The higher up one goes in the number of occupied CF Landau levels, the larger is the number of $\mathrm{CF}$ bound states with the correct quantum numbers: consequently, the overlap between the single-electron or single-hole state and and any CF bound state plummets. Similarly, in the present approach, the reduction of $Z$ is caused by the larger and larger number of magnetophonons that can "dress up" the test electron as the gap becomes smaller.

In conclusion, I have presented qualitative and quantitative arguments supporting the idea that electron (hole) quasiparticles of integral charge $e$ should exist in incompressible fractional quantum Hall liquids. This conclusion is consistent with the recent findings of Jain and Peterson. ${ }^{17}$ The new quasiparticles could be observed as conductance resonances in vertical tunneling spectroscopy, ${ }^{9}$ as discussed in Ref. 17.

Support from NSF Grant No. DMR-0313681 is gratefully acknowledged.
*Electronic address: vignaleg@missouri.edu

${ }^{1}$ G. F. Giuliani and G. Vignale, in Quantum Theory of the Electron Liquid (Cambridge University Press, Cambridge, 2005).

${ }^{2}$ The Quantum Hall Effect, edited by R. E. Prange and S. M. Girvin (Springer-Verlag, New York, 1987); Perspectives in Quantum Hall Effect, edited by S. Das Sarma and R. Pinczuk (Wiley, New York, 1990).

${ }^{3}$ D. C. Tsui, H. L. Störmer, and A. C. Gossard, Phys. Rev. Lett. 48, 1559 (1982).

${ }^{4}$ R. B. Laughlin, Phys. Rev. Lett. 50, 1395 (1983).

${ }^{5}$ D. Arovas, J. R. Schrieffer, and F. Wilczek, Phys. Rev. Lett. 53, 722 (1984).

${ }^{6}$ S. M. Girvin, A. H. MacDonald, and P. M. Platzman, Phys. Rev. B 33, 2481 (1986).

${ }^{7}$ J. K. Jain, Phys. Rev. Lett. 63, 199 (1989).

${ }^{8}$ Composite Fermions, edited by O. Heinonen (World Scientific, Singapore, 1998).

${ }^{9}$ J. P. Eisenstein, L. N. Pfeiffer, and K. W. West, Phys. Rev. Lett. 69, 3804 (1992).

${ }^{10}$ R. C. Ashoori, J. A. Lebens, N. P. Bigelow, and R. H. Silsbee, Phys. Rev. Lett. 64, 681 (1990); Phys. Rev. B 48, 4616 (1993).

${ }^{11}$ P. Johansson and J. M. Kinaret, Phys. Rev. Lett. 71, 1435 (1993);
Phys. Rev. B 50, 4671 (1994).

${ }^{12}$ S. He, P. M. Platzman, and B. I. Halperin, Phys. Rev. Lett. 71, 777 (1993).

${ }^{13}$ Y. Hatsugai, P. A. Bares, and X. G. Wen, Phys. Rev. Lett. 71, 424 (1993).

${ }^{14}$ A. L. Efros and F. G. Pikus, Phys. Rev. B 48, 14694 (1993).

${ }^{15}$ R. Haussmann, Phys. Rev. B 53, 7357 (1996).

${ }^{16}$ E. H. Rezayi, Phys. Rev. B 35, 3032 (1987).

${ }^{17}$ J. K. Jain and M. R. Peterson, Phys. Rev. Lett. 94, 186808 (2005).

${ }^{18}$ B. I. Halperin, P. A. Lee, and N. Read, Phys. Rev. B 47, 7312 (1993).

${ }^{19}$ S. Conti and G. Vignale, J. Phys.: Condens. Matter 19, L779 (1998).

${ }^{20}$ L. Bonsall and A. A. Maradudin, Phys. Rev. B 15, 1959 (1977).

${ }^{21}$ R. Asgari, B. Davoudi, M. Polini, G. F. Giuliani, M. P. Tosi, and G. Vignale, Phys. Rev. B 71, 045323 (2005).

${ }^{22}$ R. K. Kamilla and J. K. Jain, Int. J. Mod. Phys. B 11, 2621 (1997); J. K. Jain and R. K. Kamilla, Phys. Rev. B 55, R4895 (1997).

${ }^{23}$ Otherwise, using the shear modulus of the Wigner crystal, we get $E_{p}=-0.0244 \nu^{3 / 2} / \bar{\Delta}^{2}$. 